\title{
The transcription factor DNR from Pseudomonas aeruginosa specifically requires nitric oxide and haem for the activation of a target promoter in Escherichia coli
}

Correspondence

Francesca Cutruzzolà

francesca.cutruzzola@uniroma1.it

Received 4 February 2009

Revised 22 May 2009

Accepted 27 May 2009

\author{
Nicoletta Castiglione, ${ }^{1}$ Serena Rinaldo, ${ }^{1}$ Giorgio Giardina ${ }^{1}$ \\ and Francesca Cutruzzolà ${ }^{1,2}$ \\ 'Department of Biochemical Sciences 'A. Rossi Fanelli', Sapienza University of Rome, Rome, Italy \\ ${ }^{2}$ Consorzio INBB, 00136 Rome, Italy
}

\begin{abstract}
Pseudomonas aeruginosa is a well-known pathogen in chronic respiratory diseases such as cystic fibrosis. Infectivity of $P$. aeruginosa is related to the ability to grow under oxygen-limited conditions using the anaerobic metabolism of denitrification, in which nitrate is reduced to dinitrogen via nitric oxide (NO). Denitrification is activated by a cascade of redox-sensitive transcription factors, among which is the DNR regulator, sensitive to nitrogen oxides. To gain further insight into the mechanism of NO-sensing by DNR, we have developed an Escherichia coli-based reporter system to investigate different aspects of DNR activity. In E. coli DNR responds to NO, as shown by its ability to transactivate the $P$. aeruginosa norCB promoter. The direct binding of DNR to the target DNA is required, since mutations in the helix-turn-helix domain of DNR and specific nucleotide substitutions in the consensus sequence of the norCB promoter abolish the transcriptional activity. Using an E. coli strain deficient in haem biosynthesis, we have also confirmed that haem is required in vivo for the NO-dependent DNR activity, in agreement with the property of DNR to bind haem in vitro. Finally, we have shown, we believe for the first time, that DNR is able to discriminate in vivo between different diatomic signal molecules, $\mathrm{NO}$ and $\mathrm{CO}$, both ligands of the reduced haem iron in vitro, suggesting that DNR responds specifically to NO.
\end{abstract}

\section{INTRODUCTION}

Pseudomonas aeruginosa is one of the most important opportunistic pathogens; it is a Gram-negative bacterium which colonizes the inflamed lungs of cystic fibrosis patients, causing persistent infections (Yoon et al., 2006). $P$. aeruginosa is able to grow in the absence of oxygen, through anaerobic metabolism, which is important for infectivity and for the formation of biofilm (Hassett et al., 2002; Barraud et al., 2006), a surface-associated antibioticresistant microbial community (Singh et al., 2000). The molecular race between host and pathogen thus includes strategies that are centred on the ability of $P$. aeruginosa to survive under oxygen-limited conditions; cells lying near the edge of the mucous layer rapidly deplete oxygen $\left(\mathrm{O}_{2}\right)$, creating a gradient where $\mathrm{O}_{2}$ drops to very low levels. Under these microaerobic conditions $P$. aeruginosa grows in thick biofilms probably employing both microaerobic

Abbreviations: ALA, 5-aminolaevulinic acid; CRP, CAMP receptor protein; DNR, dissimilatory nitrate respiration regulator; FNR, fumarate and nitrate reductase regulator; HTH, helix-turn-helix; SNP, sodium nitroprusside; $\beta$-gal, $\beta$-galactosidase. respiration and the denitrifying redox chain (Yoon et al., 2002; Alvarez-Ortega \& Harwood, 2007; Platt et al., 2008). The complete denitrification pathway involves four enzymes: nitrate reductase, nitrite reductase, nitric oxide reductase (NOR) and nitrous oxide reductase, operating sequentially to reduce nitrate to dinitrogen gas via nitrite $\left(\mathrm{NO}_{2}^{-}\right)$, nitric oxide $(\mathrm{NO})$ and nitrous oxide $\left(\mathrm{N}_{2} \mathrm{O}\right)$ (Zumft, 1997). The expression and the activity of the NIR and NOR enzymes are tightly controlled because it is mandatory for the bacteria to keep the concentration of intracellular NO below cytotoxic levels, to limit nitrosative stress.

In $P$. aeruginosa the denitrification pathway is regulated by redox signalling, through a cascade of transcription factors; in particular, the global oxygen-sensing regulator ANR (anaerobic regulation of arginine deaminase and nitrate reduction) (Galimand et al., 1991), a homologue of the Escherichia coli oxygen sensor FNR protein, activates, under anaerobic conditions, the gene coding for the transcription factor DNR (dissimilatory nitrate respiration regulator), which, in the presence of $\mathrm{N}$-oxides, promotes the expression of the nir, the nor and the nos genes (Arai et al., 1995, 1997, 1999, 2003). 
The DNR transcription factor belongs to the CRP/FNR (cAMP receptor protein/fumarate and nitrate reductase regulator) superfamily of regulators (Zumft, 2002; Körner et al., 2003). The members of this superfamily are usually homodimers, each monomer being formed by three domains (McKay \& Steitz, 1981): (i) an N-terminal sensing domain (also referred to as the effector domain) with the typical fold of the cAMP-binding domain of CRP; (ii) a long dimerization $\alpha$-helix recruited to form the dimer interface; and (iii) a C-terminal DNA-binding domain that contains a helix-turn-helix (HTH) motif.

$\mathrm{N}$-oxide sensors belong to different subgroups of the CRP/ FNR superfamily, namely the DNR, NnrR and FNR subgroups (Rinaldo et al., 2006), which recognize the same consensus sequence in their target promoters, the FNR box (TTGATN ${ }_{4}$ ATCAA) (Eiglmeier et al., 1989; Hoeren et al., 1993; Hasegawa et al., 1998); these proteins contain a highly conserved amino acid sequence motif Glu-SerArg (E-SR), directly involved in the interaction with the FNR box (Green et al., 2001). Another transcription factor belonging to the $\mathrm{E}$ subgroup of the CRP/FNR superfamily, i.e. NssR from Campylobacter jejuni, has been recently described to act, perhaps indirectly, as a NO sensor (Elvers et al., 2005).

Previous studies, carried out in $P$. aeruginosa $\mathrm{PAO} 1$ with the nirS, the nor $C$ and the nos $Z$ promoters fused to the lac $Z$ reporter gene, showed that the DNR transcription factor responds in vivo to N-oxides (Arai et al., 1999, 2003). A similar response to $\mathrm{NO}$ in vivo has been reported for the DNR homologue NNR in Paracoccus denitrificans (Van Spanning et al., 1999) and, for the same regulator, also in $E$. coli using the FNR-dependent E. coli melR promoter (Hutchings et al., 2000).

Understanding of the biochemical mechanisms used to respond to specific redox signals (such as NO) is crucial in order to dissect the in vivo function of a sensor protein. The molecular mechanism underlying the activity of DNR is still elusive, mainly because a reliable system to probe the NO-dependent DNA binding of this regulator has not been available. Therefore to investigate in detail the NOdependent activity of $P$. aeruginosa DNR, we have studied the DNR-mediated transcriptional activity in E. coli, a species which is unable to carry out denitrification. A $\beta$ galactosidase ( $\beta$-gal) assay was performed under anaerobic conditions in the presence of $\mathrm{N}$-oxides, using the putative DNR target promoter nor $C B$ (hereinafter nor) from $P$. aeruginosa. The choice of this promoter is supported by previous evidence showing that in $P$. aeruginosa, in the presence of N-oxides, nor is more strongly activated by DNR than nirS (Arai et al., 1999). In the first part of this paper we report the successful development of this system, and the characterization of the NO-dependent activity of DNR in E. coli.

To gain further insight into the mechanism of NO-sensing by DNR or other homologous transcription factors, in the second part of the paper we describe how the system developed in E. coli was successfully used to investigate different aspects of the DNR activity. We have demonstrated the specificity of the DNR-DNA interaction, analysing both the transcriptional activity of DNR mutants and the effect of mutations in the FNR box of the nor promoter. Since spectroscopic and structural evidence and in vitro functional assays suggested the involvement of haem in DNR activity (Giardina et al., 2008), we have also confirmed the importance of this cofactor in the mechanism of DNR-mediated activation of the nor promoter in E. coli.

Finally, in order to test whether DNR responds specifically to NO, we investigated its activity in the presence of carbon monoxide $(\mathrm{CO})$.

\section{METHODS}

Plasmid construction and mutagenesis. The $d n r$ gene was cloned into the pACYc184 vector (Biolabs) under the constitutive tet promoter; an extra EcoRV restriction site and the ribosome-binding site were also added, yielding the plasmid pACY-DNR. The nor-lacZ reporter system was obtained by cloning the nor promoter from the $P$. aeruginosa PAO1 genome first into the EcoRI and BamHI sites of the pUC18 vector, yielding the plasmid pUC-NOR and subsequently into the EcoRI and BamHI sites of the pRS415 vector, yielding the plasmid pRS-NOR. The nor promoter fragment used in this work includes 150 bp upstream of the ATG of the nor $C B$ gene (Fig. 1). Site-directed mutagenesis was done using the QuikChange mutagenesis kit (Stratagene) according to the manufacturer's instructions, with pACY-DNR or pUC-NOR as a template. Two mutants were obtained in the HTH region of DNR: N152stop, previously described (Giardina et al., 2008), which lacks the entire HTH domain, and E193D, which has a substitution (Glu to Asp) in position 193. The mutations introduced in the FNR box of the nor promoter were as follows: a double mutation in which the GA bases in positions 3 and 4 have been substituted with AG (TTAGTN ${ }_{4}$ ATCAA, named norl), and a single mutation in which the A base in position 4 has been changed to a G (TTGGTN ${ }_{4}$ ATCAA, named nor 2 ). The nor 1 and nor 2 fragments were then inserted into the EcoRI and BamHI sites of the pRS415 vector, yielding, respectively, plasmids pRS-NOR1 and pRS-NOR2.

In vivo $\boldsymbol{\beta}$-galactosidase assay. Plasmids $\mathrm{pACY}-\mathrm{DNR}$ and $\mathrm{pRS}-$ NOR were transformed into E. coli TOP10 (Invitrogen) $\left[\mathrm{F}^{-}\right.$mcrA $\Delta$ (mrr-hsdRMS-mcrBC) $\phi 80$ lacZLM15 $\Delta$ lacX74 nupG recA1 araD139 $\Delta($ ara-leu $) 7697$ galE15 galK16 $\operatorname{rpsL}\left(\mathrm{Str}^{\mathrm{R}}\right)$ endA1 $\left.\lambda^{-}\right]$or E. coli C600 $\left[\mathrm{F}^{-}\right.$tonA21 thi-1 thr-1 leuB6 lacY1 glnV44 rfbC1 fhuA1 $\lambda^{-}$ (hemA::kan)] ( $\triangle$ hemA) (a kind gift of Cécile Wandersman, Institut Pasteur, Paris, France). The TOP10 strain is phenotypically $\mathrm{FNR}^{+}$, as tested in a control experiment with an FNR-dependent reporter fusion (pRW50 carrying the FF-pmelR fragment) (Wing et al., 1995) (data not shown). The rich medium for bacterial growth was LuriaBertani (LB) broth [tryptone $\left(10 \mathrm{~g} \mathrm{l}^{-1}\right)$, yeast extract $\left(5 \mathrm{~g} \mathrm{l}^{-1}\right), \mathrm{NaCl}$ $\left(10 \mathrm{~g} \mathrm{l}^{-1}\right)$ ], supplemented with $0.4 \%(\mathrm{w} / \mathrm{v})$ glucose (LG broth) and $0.4 \mathrm{mM}$ ALA (5-aminolaevulinic acid hydrochloride), as indicated.

DNR activity was tested by the $\beta$-gal assay at least in duplicate according to the method of Miller (1972) on at least two independently grown cultures. The aerobic cultures were grown in $50 \mathrm{ml}$ medium in $250 \mathrm{ml}$ flasks at $37{ }^{\circ} \mathrm{C}$ to $\mathrm{OD}_{600} 0.4$ and were shaken at 200 r.p.m. Anaerobic growth was carried out by transferring $10 \mathrm{ml}$ of the aerobic samples from the flasks to filled tubes sealed with a rubber cap. After $30 \mathrm{~min}$ of growth to remove the residual oxygen, selected samples were induced with either $10 \mu \mathrm{M}$ 
1

Fig. 1. Sequence of the $P$. aeruginosa nor promoter fragment used in this study ( $150 \mathrm{bp})$. The putative binding site for the DNR protein is boxed and the bases of the consensus FNR box are highlighted in bold; the initial part of the coding sequence of the norCB gene, not included in the PCR fragment, is shown in grey and the translational start point is underlined.

aqueous NO from a saturated solution $(2 \mathrm{mM})$ or $2 \mathrm{mM}$ nitrite using a gas-tight syringe or, if indicated, with $5 \%(\mathrm{v} / \mathrm{v}) \mathrm{CO}$ gas; the cultures were then incubated without shaking for $1 \mathrm{~h}$ before $\beta$-gal was assayed. For the N152stop mutant, the growth temperature was switched from $37{ }^{\circ} \mathrm{C}$ to $20{ }^{\circ} \mathrm{C}$ after $1 \mathrm{~h}$ of growth. For the $\Delta$ hemA strain, the rich medium was supplemented with ALA when indicated.

For Western blot analysis, cultures were grown under the same conditions as used for the $\beta$-galactosidase assays; cells harvested and disrupted by boiling in the loading buffer $(1.50 \mathrm{mM}$ Tris/ $\mathrm{HCl} \mathrm{pH} 6.8$, 300 mM DTT, $6 \%$ SDS, $0.3 \%$ bromophenol blue, $30 \%$, v/v, glycerol) were separated by SDS-PAGE and Western blot analysis was carried out. The DNR protein was detected using a polyclonal rabbit antibody (Davids Biotechnologies) and a goat anti-rabbit IgG (Sigma) alkaline phosphatase-conjugated secondary antibody.

\section{RESULTS AND DISCUSSION}

\section{In vivo activation of DNR by $\mathrm{N}$-oxides in the heterologous $E$. coli system}

The effect of $\mathrm{N}$-oxides on the activation by DNR of the nir, nor and nos genes has been previously demonstrated in $P$. aeruginosa (Arai et al., 1999, 2003). The primary aim of our work was to characterize the NO-dependent DNR transcriptional activity in a heterologous model organism such as E. coli, which is unable to carry out denitrification. Plasmid pRS-NOR, carrying the $P$. aeruginosa nor promoter (Fig. 1) was transformed into E. coli TOP10, with or without plasmid pACY-DNR, harbouring the $d n r$ gene under the control of a constitutive tet promoter. A $\beta$-gal assay according to the method of Miller (1972) was carried out under aerobic and anaerobic conditions in the presence of different $\mathrm{N}$-oxides.

As shown in Fig. 2, DNR was able to transactivate the nor promoter in E. coli under anaerobic conditions and in the presence of $10 \mu \mathrm{M}$ NO. The maximal activation of the nor promoter was observed at $10 \mu \mathrm{M} \mathrm{NO}$, testing a range of NO concentrations from $5 \mu \mathrm{M}$ to $50 \mu \mathrm{M}$; the increase of the NO concentration from $10 \mu \mathrm{M}$ to $50 \mu \mathrm{M}$ had no influence on the cell growth but slightly decreased the DNR activation (data not shown). An activation of the nor promoter under anaerobic conditions comparable to that obtained with $\mathrm{NO}$ gas was observed using $100 \mu \mathrm{M}$ sodium nitroprusside (SNP), a nitrosylating agent and a source of $\mathrm{NO}^{+}$(data not shown).
The activation observed in the presence of $2 \mathrm{mM}$ nitrite (Fig. 2) can be explained since it is known that, in E. coli, nitrite is a source of $\mathrm{NO}$, due to the activity of nitrite reductase, which reduces nitrite to ammonia (Corker \& Poole, 2003). Nitrite is not directly involved in the activation of DNR since no activity was observed in air in the presence of $2 \mathrm{mM}$ nitrite, a condition in which $\mathrm{NO}$ is not produced (Darwin et al., 1993).

As shown in Fig. 2, DNR is unable to activate the nor promoter under aerobic conditions. We also tested the

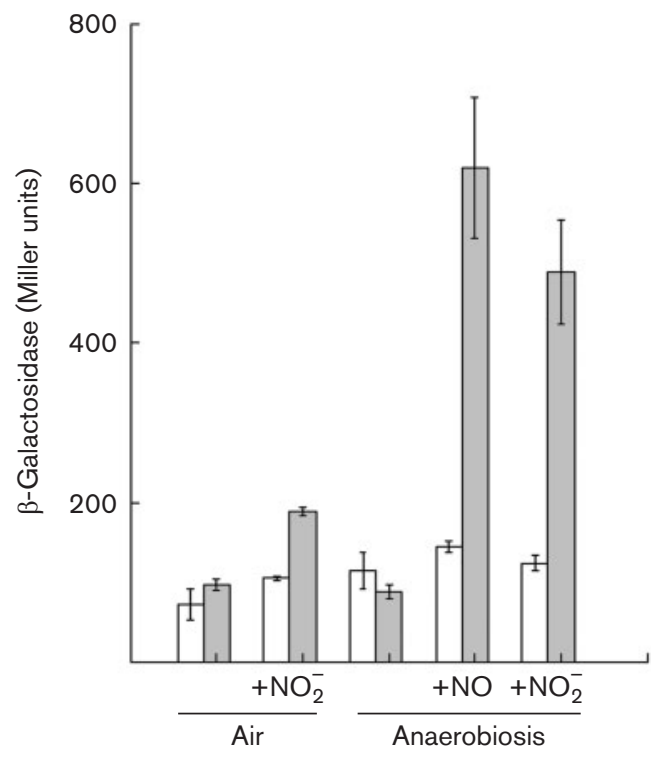

Fig. 2. Activation of the $P$. aeruginosa nor promoter requires DNR and $\mathrm{N}$-oxides. The DNR protein was expressed constitutively in $E$. coli TOP10 containing plasmid pRS-NOR, in which the nor promoter is fused to lacZ (grey bars). As a control, the $\beta$ galactosidase activity was assayed under the same experimental conditions in E. coli TOP10 containing only the pRS-NOR vector (white bars). Cultures were grown aerobically and anaerobically in LG medium to exponential phase, and induced, as indicated, by the addition of $10 \mu \mathrm{M} \mathrm{NO}$ or $2 \mathrm{mM}$ sodium nitrite. $\beta$-Galactosidase activity was measured $1 \mathrm{~h}$ after induction in duplicate in three independently grown cultures; mean values are shown, together with experimental error bars (SD). 
activity of the protein in air in the presence of SNP; the use of SNP as a source of $\mathrm{NO}^{+}$under aerobic conditions has been previously reported for the NorR protein (Hutchings et al., 2002). No DNR activity was observed in air in the presence of SNP (data not shown), in agreement with the physiological role of DNR, which, in $P$. aeruginosa, is involved in the regulation of denitrification, an alternative respiratory metabolism activated under oxygen-limiting conditions (Zumft, 1997).

Our results show that DNR is able to activate its target promoter in E. coli, suggesting that the transcriptional machinery of this bacterium can be efficiently used with a $P$. aeruginosa promoter such as the nor promoter. A similar NO-dependent transactivation in E. coli was obtained with the NNR protein from Paracoccus denitrificans; in the latter case, however, an FNR-dependent E. coli promoter was used (Hutchings et al., 2000).

The nor promoter fragment used in this study includes $150 \mathrm{bp}$ upstream of the ATG of the nor $C B$ gene; this fragment contains the canonical recognition motif for the transcriptional activator protein FNR, named the FNR box, centred -64.5 bases upstream of the ATG (Fig. 1). Interestingly, no activation of the nor promoter was observed in the TOP10 strain containing only plasmid pRS-NOR under anaerobic conditions (Fig. 2), suggesting that the endogenous FNR protein is unable to recognize this promoter. A preliminary in silico analysis of the $P$. aeruginosa nor promoter (identifying a good candidate for a -10 sequence centred around base 119.5) and its comparison with the norC promoter of Pseudomonas stutzeri (in which the transcriptional start site has been mapped: Zumft et al., 1994) suggest that the FNR box could be located as in class II promoters; this hypothesis will require to be further confirmed experimentally. Interestingly, however, the region located between the palindromic TTGAT motif of the consensus FNR box in the nor promoter has a high GC content (Fig. 1), possibly explaining the lack of activation, given that it is known that FNR favours an AT-rich motif against a GC-rich motif in this region (Scott et al., 2003). We cannot exclude, however, that other features of the nor promoter may preclude productive interaction between FNR and the E. coli transcriptional machinery. Interestingly, although the E. coli FNR protein seems unable to activate the nor promoter (this work), the DNR protein is able to recognize in $P$. aeruginosa an FNR-dependent $E$. coli promoter (Hasegawa et al., 1998), making the scenario more complicated.

\section{DNR binds specifically the nor promoter}

To demonstrate the specificity of the DNR-nor promoter interaction in the E. coli system described above, we performed the $\beta$-gal assay with different mutants which have mutations in the HTH DNA recognition motif of the DNR protein or in the FNR box of the nor promoter.
The inability of the N152stop mutant, which lacks the last 75 amino acids and hence the entire DNA-binding domain of DNR (Giardina et al., 2008), to transactivate the nor promoter under anaerobiosis in the presence of NO (Fig. 3a) suggests that the NO-dependent activation of the nor promoter requires the direct binding of DNR to the target DNA. To further analyse the DNR-DNA interaction, we focused our attention on the Glu-193 residue of the protein, a position highly conserved in the CRP/FNR superfamily (Green et al., 2001); this glutamate residue was shown to be involved in the direct interaction of the FNR protein with the FNR box sequence on the target promoter (Spiro et al., 1990). In agreement with the predicted involvement of this glutamate in binding the FNR box, the Glu 193 to Asp (E193D) variant of the DNR protein was unable to activate the nor promoter (Fig. 3a) under anaerobiosis in the presence of NO. This result confirms the specificity of the interaction of DNR with the nor promoter. The lack of activation by the two DNR mutants in the E. coli strain is not due to the lack of expression of the mutated proteins, which are correctly produced in the bacterium as verified by Western blotting (data not shown).

We also probed the DNR-DNA interaction by producing specific base substitutions in the FNR box of the nor promoter. Two mutants were produced: the first one carries an inversion of two bases in the FNR box (TTAGT$\mathrm{N}_{4}$ TTGAT) (norl) and the second one carries a single base change $\left(\mathrm{A}_{4}\right.$ to $\mathrm{G}$ ) (TTGGTN ${ }_{4} \mathrm{TTGAT}$ ) (nor 2 ). The corresponding plasmids (see Methods) were transformed into an E. coli strain harbouring the plasmid pACYDNR and the $\beta$-gal activity under anaerobic conditions with or without NO gas was measured. As shown in Fig. 3(b), DNR was unable to activate both mutant promoters under anaerobiosis in the presence of NO. These results confirm again the specificity of the interaction between DNR and the target DNA in the E. coli system. Moreover we have demonstrated, to our knowledge for the first time, that a single nucleotide substitution in the FNR box is sufficient to completely disrupt the activity of the DNR protein on the nor promoter. The sensitivity of the FNR box to the $\mathrm{A}_{4}$ to $G$ mutation in the consensus sequence was shown also for the narGHJI promoter, an E. coli FNR target (Walker \& DeMoss, 1992).

\section{DNR activity requires haem biosynthesis}

The mechanism whereby DNR can sense the signal molecule is not yet understood; it is certain, however, that DNR, which lacks conserved cysteines, is unable to form an $\mathrm{Fe}-\mathrm{S}$ centre that can interact with the signal molecule as reported for the $\mathrm{O}_{2}$ sensor FNR (Khoroshilova et al., 1995, 1997; Green et al., 1996; Jordan et al., 1997). Moreover, spectroscopic evidence supports the hypothesis that haem is involved in NO sensing; in fact the DNR-haem complex is stable and can bind NO in the ferrous state to yield a pentacoordinate derivative (Giardina et al., 2008). In 

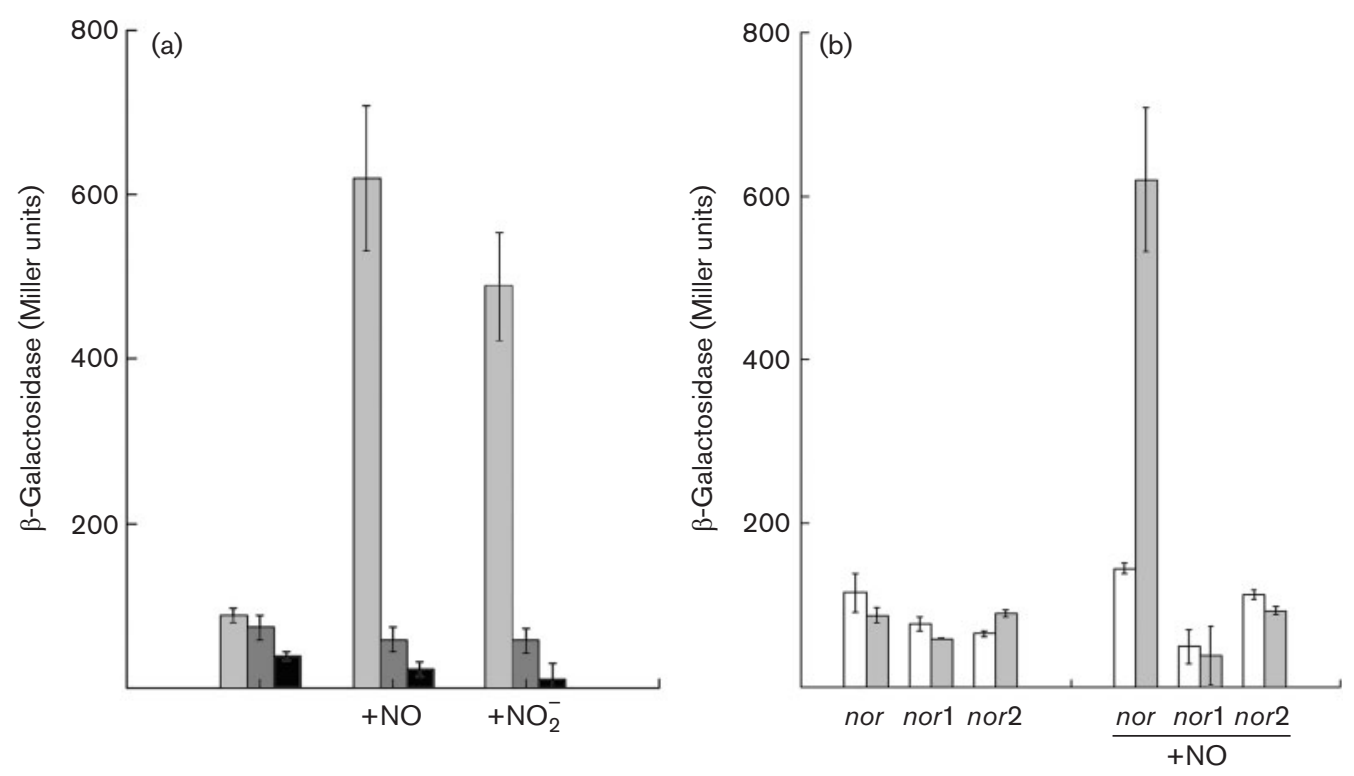

Fig. 3. Activation of the nor promoter requires the direct binding of DNR. (a) The N152stop protein, lacking the DNA-binding domain (dark grey bars) or the E193D protein (black bars) was expressed constitutively in E. coli TOP10 together with plasmid $\mathrm{pRS}-\mathrm{NOR}$ and the $\beta$-galactosidase activity was measured. For comparison, the $\beta$-galactosidase activity of the wild-type protein is also shown (grey bars). (b) Plasmid pRS415 containing the wild-type nor promoter or the mutant promoters nor1 (FNR box: TTAGTN $_{4}$ ATCAA) or nor2 (FNR box: TTGGTN ${ }_{4}$ ATCAA) was expressed constitutively in E. coli TOP10 together with pACYDNR (grey bars). As a control, the $\beta$-galactosidase activity was measured under the same experimental conditions in $E$. coli TOP10 containing only the plasmids with the wild-type and mutant promoters (white bars). Cultures were grown anaerobically in LG medium to exponential phase, and induced, as indicated, by the addition of $10 \mu \mathrm{M} \mathrm{NO}$. $\beta$-Galactosidase activity was measured $1 \mathrm{~h}$ after induction in duplicate in three independently grown cultures; mean values are shown, together with experimental error bars (SD).

addition, the only evidence in the literature in which a member of the DNR class of regulators binds the target DNA in vitro was obtained by an electophoretic mobility shift assay using the $P$. aeruginosa DNR protein bound to haem (Giardina et al., 2008). The hypothesis of a haembased sensing is reasonable also in the light of a similar haem-based mechanism well known for the Rhodospirillum rubrum CooA protein (Shelver et al., 1997); in this case the $\mathrm{CO}$ molecule binds to the haem iron and triggers a conformational change, thus regulating the transcriptional activity (Lanzilotta et al., 2000). These findings suggest that a haem-based mechanism is the most likely to explain the NO-dependent regulation of the function of DNR.

To demonstrate that the haem is required for DNR activity in vivo, we measured the NO-dependent activation of the nor promoter in a haem-deficient $(\Delta h e m A)$ E. coli strain, which lacks the HemA protein involved in the production of ALA, a key intermediate in the biosynthesis of haem (Harris et al., 1993). A similar approach was successfully employed to show that haem biosynthesis is essential for the sensing activity of NNR from Paracoccus denitrificans (Lee et al., 2006).

Plasmid pRS-NOR was transformed into the E. coli $\Delta$ hemA strain with or without plasmid pACY-DNR, and cells were grown in the presence or in the absence of $0.4 \mathrm{mM}$ ALA, under anaerobiosis with or without NO. To evaluate the basal activity of the nor promoter, we also measured the $\beta$ gal activity in the E. coli $\Delta$ hemA strain containing only plasmid pRS-NOR.

The results (Fig. 4) show that only in the presence of exogenous ALA is a NO-dependent DNR activity comparable to that seen in the TOP10 strain observed; this demonstrates that in E. coli haem is required to support the activation of the nor promoter by DNR in vivo, confirming previous evidence obtained in vitro.

\section{DNR discriminates between different diatomic gases}

Given that haem is essential for DNR activation we tested whether DNR is able to discriminate between different gases. We have previously shown that the reduced form of the DNR-haem complex is able to bind both $\mathrm{NO}$ and $\mathrm{CO}$ in vitro (Giardina et al., 2008). In particular, the adduct of NO with the haem-DNR complex is pentacoordinate, whereas the CO-bound species is hexacoordinate. Therefore, we used the E. coli system developed here to carry out a $\beta$-gal assay under anaerobic conditions in the 


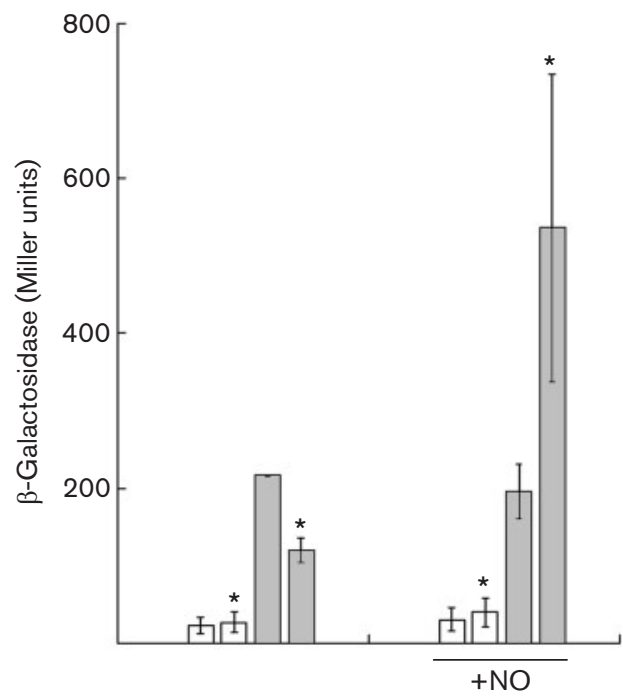

Fig. 4. Haem dependence of DNR-mediated $\beta$-galactosidase activity. The DNR protein was expressed constitutively in the $\Delta$ hemA E. coli strain together with plasmid pRS-NOR (grey bars). As a control, the $\beta$-galactosidase activity was assayed under the same experimental conditions in the $\Delta h e m A E$. coli strain containing only the pRS-NOR vector (white bars). Cultures were grown anaerobically in LG medium to exponential phase, and induced, as indicated, by the addition of $10 \mu \mathrm{M} N O$; samples marked with asterisk on the graph refer to cultures grown in the presence of $0.4 \mathrm{mM}$ ALA. $\beta$-Galactosidase activity (Miller, 1972) was measured $1 \mathrm{~h}$ after induction (grey bars) in duplicate in three independently grown cultures; mean values (Miller units) are shown, together with experimental error bars (SD).

presence of CO. The very low ( $25.5 \pm 3$ Miller units) $\beta$-gal activity measured under these conditions shows that DNR is inactive in the presence of $\mathrm{CO}$, suggesting that the protein responds differently to the two diatomic signal molecules, i.e. NO and CO. These results are in agreement with our previous in vitro evidence that the haem coordination state of the NO and CO complexes in DNR is different (Giardina et al., 2008). In conclusion, NO is the specific and unique signal required for DNR function; this selective activation in response to a particular ligand is shared with other haem-based gas sensors (e.g. CooA and H-NOX domains) (Andrew et al., 2001; Boon \& Marletta, 2005; Gilles-Gonzalez \& Gonzalez, 2005).

\section{Conclusions}

We have shown in this work that the $P$. aeruginosa DNR protein is active in a heterologous system (E. coli) and can use the transcriptional apparatus of this bacterium to bind specifically to the $P$. aeruginosa nor promoter in order to regulate its activation. Moreover, we have shown that haem is the cofactor mediating the NO-dependent DNR regulation in E. coli; this finding reinforces the in vitro results previously obtained by our group. We have demonstrated, we believe for the first time, that DNR is able to discriminate between different diatomic signal molecules, $\mathrm{NO}$ and $\mathrm{CO}$.

We can therefore conclude that the heterologous system described has shed new light on the features of the $P$. aeruginosa DNR protein and, in the future, will allow us to assay the activity of site-directed mutants targeting residues putatively involved in haem binding and in NO-sensing.

\section{ACKNOWLEDGEMENTS}

We wish to thank Maurizio Brunori (Rome, Italy) for critical reading of the manuscript. We also thank Manuela Caruso (Rome, I) for skilful technical assistance and Cécile Wandersman (Institut Pasteur, Paris, France) for providing the $\Delta$ hemA E. coli bacterial strain. The work was supported by funds from Ministero della Università e della Ricerca of Italy (RBIN04PWN_000; RBIN04PWN_002; 20074TJ3ZB_005).

\section{REFERENCES}

Alvarez-Ortega, C. \& Harwood, C. S. (2007). Responses of Pseudomonas aeruginosa to low oxygen indicate that growth in the cystic fibrosis lung is by aerobic respiration. Mol Microbiol 65, 153-165.

Andrew, C. R., Green, E. L., Lawson, D. M. \& Eady, R. R. (2001). Resonance Raman studies of cytochrome $c^{\prime}$ support the binding of $\mathrm{NO}$ and $\mathrm{CO}$ to opposite sides of the heme: implications for ligand discrimination in heme-based sensors. Biochemistry 40, 4115-4122.

Arai, H., Igarashi, Y. \& Kodama, T. (1995). Expression of the nir and nor genes for denitrification of Pseudomonas aeruginosa requires a novel CRP/FNR-related transcriptional regulator, DNR, in addition to ANR. FEBS Lett 371, 73-76.

Arai, H., Kodama, T. \& Igarashi, Y. (1997). Cascade regulation of the two CRP/FNR-related transcriptional regulators (ANR and DNR) and the denitrification enzymes in Pseudomonas aeruginosa. Mol Microbiol 25, 1141-1148.

Arai, H., Kodama, T. \& Igarashi, Y. (1999). Effect of nitrogen oxides on expression of the nir and nor genes for denitrification in Pseudomonas aeruginosa. FEMS Microbiol Lett 170, 19-24.

Arai, H., Mizutani, M. \& Igarashi, Y. (2003). Transcriptional regulation of the nos genes for nitrous oxide reductase in Pseudomonas aeruginosa. Microbiology 149, 29-36.

Barraud, N., Hassett, D. J., Hwang, S. H., Rice, S. A., Kjelleberg, S. \& Webb, J. S. (2006). Involvement of nitric oxide in biofilm dispersal of Pseudomonas aeruginosa. J Bacteriol 188, 7344-7353.

Boon, E. M. \& Marletta, M. A. (2005). Ligand discrimination in soluble guanylate cyclase and the H-NOX family of heme sensor proteins. Curr Opin Chem Biol 9, 441-446.

Corker, H. \& Poole, R. K. (2003). Nitric oxide formation by Escherichia coli. Dependence on nitrite reductase, the NO-sensing regulator Fnr, and flavohemoglobin Hmp. J Biol Chem 278, 31584-31592.

Darwin, A., Hussain, H., Griffiths, L., Grove, J., Sambongi, Y., Busby, S. \& Cole, J. (1993). Regulation and sequence of the structural gene for cytochrome $c_{552}$ from Escherichia coli: not a hexahaem but a 50 kDa tetrahaem nitrite reductase. Mol Microbiol 9, 1255-1265.

Eiglmeier, K., Honoré, N., luchi, S., Lin, E. C. \& Cole, S. T. (1989). Molecular genetic analysis of FNR-dependent promoters. Mol Microbiol 3, 869-878.

Elvers, K. T., Turner, S. M., Wainwright, L. M., Marsden, G., Hinds, J., Cole, J. A., Poole, R. K., Penn, C. W. \& Park, S. F. (2005). NssR, a 
member of the Crp-Fnr superfamily from Campylobacter jejuni, regulates a nitrosative stress-responsive regulon that includes both a single-domain and a truncated haemoglobin. Mol Microbiol 57, 735-750.

Galimand, M., Gamper, M., Zimmermann, A. \& Haas, D. (1991). Positive FNR-like control of anaerobic arginine degradation and nitrate respiration in Pseudomonas aeruginosa. J Bacteriol 173, 1598-1606.

Giardina, G., Rinaldo, S., Johnson, K. A., Di Matteo, A., Brunori, M. \& Cutruzzolà, F. (2008). NO sensing in Pseudomonas aeruginosa: structure of the transcriptional regulator DNR. J Mol Biol 378, 1002-1015.

Gilles-Gonzalez, M. A. \& Gonzalez, G. (2005). Heme-based sensors: defining characteristics, recent developments, and regulatory hypotheses. J Inorg Biochem 99, 1-22.

Green, J., Bennett, B., Jordan, P., Ralph, E. T., Thomson, A. J. \& Guest, J. R. (1996). Reconstitution of the [4Fe-4S] cluster in FNR and demonstration of the aerobic-anaerobic transcription switch in vitro. Biochem J 316, 887-892.

Green, J., Scott, C. \& Guest, J. R. (2001). Functional versatility in the CRP-FNR superfamily of transcription factors: FNR and FLP. Adv Microb Physiol 44, 1-34.

Harris, W. F., Burkhalter, R. S., Wen, L. \& Timkovich, R. (1993). Enhancement of bacterial porphyrin biosynthesis by exogenous aminolevulinic acid and isomer specificity of the products. Bioorg Chem 21, 209-220.

Hasegawa, N., Arai, H. \& Igarashi, Y. (1998). Activation of a consensus FNR-dependent promoter by DNR of Pseudomonas aeruginosa in response to nitrite. FEMS Microbiol Lett 166, 213-217.

Hassett, D. J., Cuppoletti, J., Trapnell, B., Lymar, S. V., Rowe, J. J., Yoon, S. S., Hilliard, G. M., Parvatiyar, K., Kamani, M. C. \& other authors (2002). Anaerobic metabolism and quorum sensing by Pseudomonas aeruginosa biofilms in chronically infected cystic fibrosis airways: rethinking antibiotic treatment strategies and drug targets. Adv Drug Deliv Rev 54, 1425-1443.

Hoeren, F. U., Berks, B. C., Ferguson, S. J. \& McCarthy, J. E. (1993). Sequence and expression of the gene encoding the respiratory nitrousoxide reductase from Paracoccus denitrificans. New and conserved structural and regulatory motifs. Eur J Biochem 218, 49-57.

Hutchings, M. I., Shearer, N., Wastell, S., van Spanning, R. J. \& Spiro, S. (2000). Heterologous NNR-mediated nitric oxide signaling in Escherichia coli. J Bacteriol 182, 6434-6439.

Hutchings, M. I., Mandhana, N. \& Spiro, S. (2002). The NorR protein of Escherichia coli activates expression of the flavorubredoxin gene norV in response to reactive nitrogen species. J Bacteriol 184, 4640-4643.

Jordan, P. A., Thomson, A. J., Ralph, E. T., Guest, J. R. \& Green, J. (1997). FNR is a direct oxygen sensor having a biphasic response curve. FEBS Lett 416, 349-352.

Khoroshilova, N., Beinert, H. \& Kiley, P. (1995). Association of a polynuclear iron-sulfur center with a mutant FNR protein enhances DNA binding. Proc Natl Acad Sci U S A 92, 2499-2503.

Khoroshilova, N., Popescu, C., Münck, E., Beinert, H. \& Kiley, P. (1997). Iron-sulfur cluster disassembly in the FNR protein of Escherichia coli by $\mathrm{O}_{2}$ : [4Fe-4S] to [2Fe-2S] conversion with loss of biological activity. Proc Natl Acad Sci U S A 94, 6087-6092.

Körner, H., Sofia, H. J. \& Zumft, W. G. (2003). Phylogeny of the bacterial superfamily of Crp-Fnr transcription regulators: exploiting the metabolic spectrum by controlling alternative gene programs. FEMS Microbiol Rev 27, 559-592.

Lanzilotta, W. N., Schuller, D. J., Thorsteinsson, M. V., Kerby, R. L., Roberts, G. P. \& Poulos, T. L. (2000). Structure of the CO sensing transcription activator CooA. Nat Struct Biol 7, 876-880.

Lee, Y. Y., Shearer, N. \& Spiro, S. (2006). Transcription factor NNR from Paracoccus denitrificans is a sensor of both nitric oxide and oxygen: isolation of $n n r^{*}$ alleles encoding effector-independent proteins and evidence for a haem-based sensing mechanism. Microbiology 152, 1461-1470.

McKay, D. B. \& Steitz, T. A. (1981). Structure of catabolite gene activator protein at $2.9 \AA$ resolution suggests binding to left-handed B-DNA. Nature 290, 744-749.

Miller, J. H. (1972). Experiments in Molecular Genetics. Cold Spring Harbor, NY: Cold Spring Harbor Laboratory.

Platt, M. D., Schurr, M. J., Sauer, K., Vazquez, G., Kukavica-Ibrulj, I., Potvin, E., Levesque, R. C., Fedynak, A., Brinkman, F. S. \& other authors (2008). Proteomic, microarray, and signature-tagged mutagenesis analyses of anaerobic Pseudomonas aeruginosa at $\mathrm{pH} 6.5$, likely representing chronic, late-stage cystic fibrosis airway conditions. J Bacteriol 190, 2739-2758.

Rinaldo, S., Giardina, G., Brunori, M. \& Cutruzzola, F. (2006). Noxide sensing and denitrification: the DNR transcription factors. Biochem Soc Trans 34, 185-187.

Scott, C., Partridge, J. D., Stephenson, J. R. \& Green, J. (2003). DNA target sequence and FNR-dependent gene expression. FEBS Lett 541, 97-101.

Shelver, D., Kerby, R. L., He, Y. \& Roberts, G. P. (1997). CooA, a COsensing transcription factor from Rhodospirillum rubrum, is a CObinding heme protein. Proc Natl Acad Sci U S A 94, 11216-11220.

Singh, P. K., Schaefer, A. L., Parsek, M. R., Moninger, T. O., Welsh, M. J. \& Greenberg, E. P. (2000). Quorum-sensing signals indicate that cystic fibrosis lungs are infected with bacterial biofilms. Nature 407, 762-764.

Spiro, S., Gaston, K. L., Bell, A. I., Roberts, R. E., Busby, S. J. \& Guest, J. R. (1990). Interconversion of the DNA-binding specificities of two related transcription regulators, CRP and FNR. Mol Microbiol 4, 1831-1838.

Van Spanning, R. J., Houben, E., Reijnders, W. N., Spiro, S., Westerhoff, H. V. \& Saunders, N. (1999). Nitric oxide is a signal for NNR-mediated transcription activation in Paracoccus denitrificans. J Bacteriol 181, 4129-4132.

Walker, M. S. \& DeMoss, J. A. (1992). Role of alternative promoter elements in transcription from the nar promoter of Escherichia coli. J Bacteriol 174, 1119-1123.

Wing, H. J., Williams, S. M. \& Busby, S. J. (1995). Spacing requirements for transcription activation by Escherichia coli FNR protein. J Bacteriol 177, 6704-6710.

Yoon, S. S., Hennigan, R. F., Hilliard, G. M., Ochsner, U. A., Parvatiyar, K., Kamani, M. C., Allen, H. L., DeKievit, T. R., Gardner, P. R. \& other authors (2002). Pseudomonas aeruginosa anaerobic respiration in biofilms: relationships to cystic fibrosis pathogenesis. Dev Cell 3, 593-603.

Yoon, S. S., Coakley, R., Lau, G. W., Lymar, S. V., Gaston, B., Karabulut, A. C., Hennigan, R. F., Hwang, S. H., Buettner, G. \& other authors (2006). Anaerobic killing of mucoid Pseudomonas aeruginosa by acidified nitrite derivatives under cystic fibrosis airway conditions. J Clin Invest 116, 436-446.

Zumft, W. G. (1997). Cell biology and molecular basis of denitrification. Microbiol Mol Biol Rev 61, 533-616.

Zumft, W. G. (2002). Nitric oxide signaling and NO dependent transcriptional control in bacterial denitrification by members of the FNR-CRP regulator family. J Mol Microbiol Biotechnol 4, 277-286.

Zumft, W. G., Braun, C. \& Cuypers, H. (1994). Nitric oxide reductase from Pseudomonas stutzeri. Primary structure and gene organization of a novel bacterial cytochrome bc complex. Eur J Biochem 219, 481490.

Edited by: R. J. M. van Spanning 